\title{
A Patient with Recurrent Abdominal Pain and Intestinal Obstruction
}

\section{Jung Wan Han, Hyun Joo Jang}

Division of Gastroenterology, Department of Internal Medicine, Dongtan Sacred Heart Hospital, Hallym University College of Medicine, Hwasung, Korea
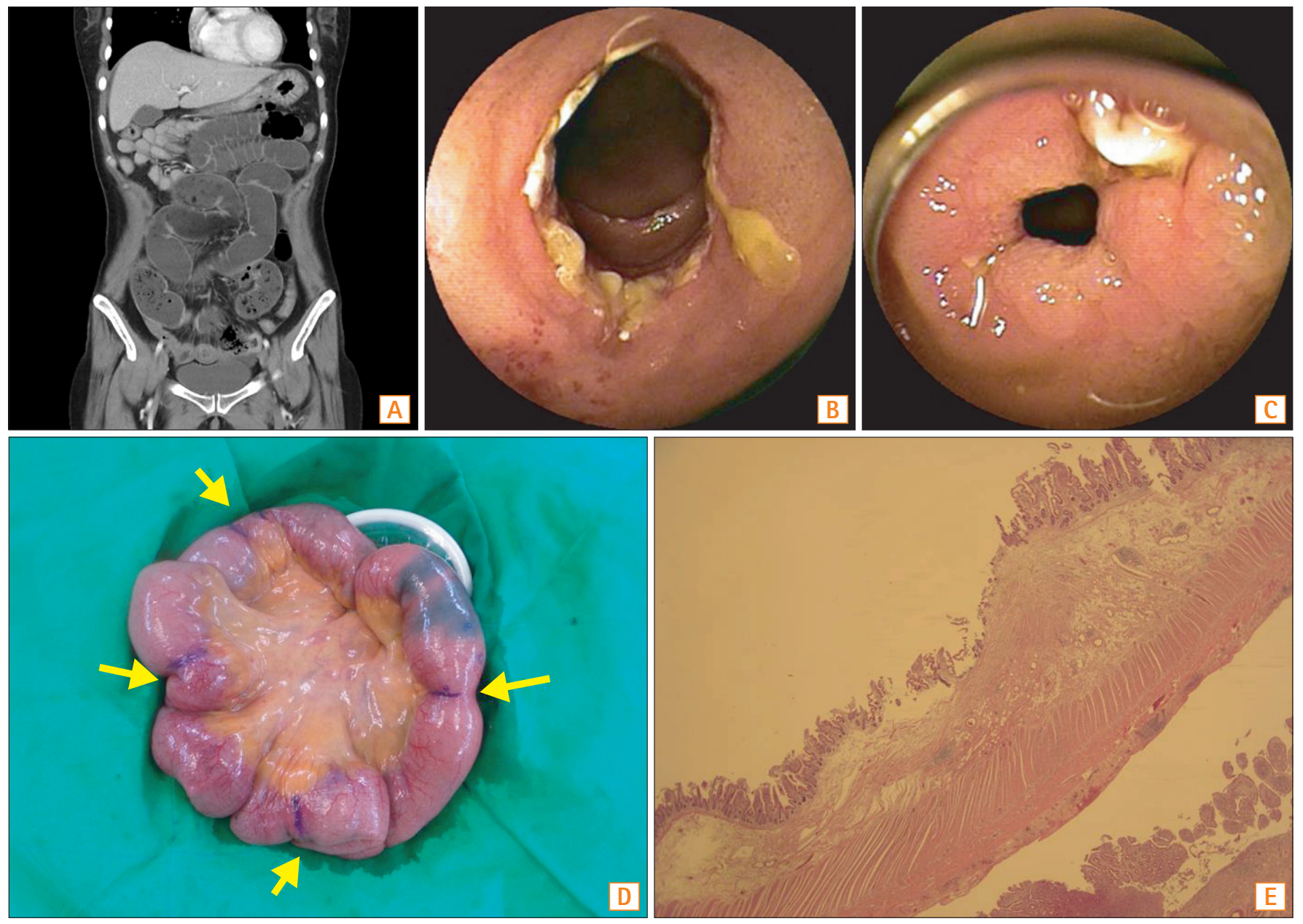

Received February 14, 2014. Revised February 14, 2014

Accepted February 14, 2014.

Correspondence to Hyun Joo Jang, Division of Gastroenterology,

Department of Internal Medicine, Dongtan Sacred Heart Hospital, Hallym

University College of Medicine, 7 Keunjaebong-gil, Hwaseong 445-907,

Korea. Tel: +82-31-8086-2450, Fax: +82-31-8086-2029, E-mail: jhj1229@

hallym.or.kr

Financial support: None. Conflict of interest: None.
Question: A 54-year-old woman was admitted to our hospital with severe recurrent abdominal pain. She had been admitted to another hospital for small intestinal obstruction with severe cramping and abdominal pain one or twice during the past year. CT at the time of the previous admissions

(c) Copyright 2014. Korean Association for the Study of Intestinal Diseases. All rights reserved.

This is an Open Access article distributed under the terms of the Creative Commons Attribution Non-Commercial License (http://creativecommons.org/licenses/by-nc/3.0) which permits unrestricted non-commercial use, distribution, and reproduction in any medium, provided the original work is properly cited. 
showed multiple small intestinal strictures, she had refused further evaluation or treatment. However, she visited our hospital after experiencing severe abdominal pain and vomiting for 2 days for evaluation of her small intestinal lesions.

Colonoscopy showed fibrous scars on the cecum and ileocecal valve, which seemed to be old scars due to tuberculous colitis. However, she had never taken any anti-tuberculosis medications and had no past history of pulmonary tuberculosis. The CT scan showed multifocal thickening, enhancement, and dilatation of small bowel (Fig. A). After decompression with a Miller-Abbott tube, doble-balloon enteroscopy (DBE) was performed, showing multiple circular ulcers with luminal narrowing in the distal jejunum (Fig. B). Enteroscopic biopsies showed chronic non-specific inflammation without granulomas. She was negative for both tuberculosis-specific Ag-induced interferon-gamma and anti-Saccharomyces cerevisiae antibodies. Since multifocal circular ulcers in the distal jejunum and colonic tuberculous scars were observed, we recommended that she begin empirical anti-tuberculosis medications with isoniazid, rifampin, ethambutol, and pyrazinamide.

The multiple ulcerative lesions improved slightly, but the ulcers with strictures remained on DBE 2 months later (Fig. C). We recommended segmental resection of the small intestinal strictures for treatment and confirmation of the pathologic diagnosis. Laparoscopic small intestinal resection with side-to-side anastomosis was performed from the distal jejunum to the proximal ileum. The gross specimen showed 4 narrowed portions with tattooing (Fig. D, yellow arrows). Microscopic findings showed ulcers with fibrinous exudates extending to the submucosa (Fig. E. H\&E, $\times 40$ ). No villous atrophy, lymphocytic vasculitis, microorganisms, giant cell granulomas, cytomegalopathy, or viral inclusions were observed. What is the most likely diagnosis?

\section{Answer to the Images: Cryptogenic Multifocal Ulcerous Stenosing Enteritis}

Cryptogenic multifocal ulcerous stenosing enteritis (CMUSE) is a rare small bowel disease characterized by chronic and recurrent bouts due to multiple small intestinal strictures and superficial ulceration affecting the mucosa and submucosa. It is an independent entity characterized by steroid-sensitive inflammation of the small bowel, which often recurs after surgery. ${ }^{1}$ However, the etiology of CMUSE remains to be clarified and its pathogenesis is poorly understood. ${ }^{2}$ Clinical features of CMUSE include chronic or relapsing ileus episodes resulting from multiple small intestinal strictures and chronic iron-deficiency anemia due to small intestinal occult blood loss. ${ }^{3}$

Diagnosis of CMUSE is based on history, clinical features, CT or magnetic resonance enteroclysis, small intestinal endoscopy, and histology of the small bowel. With the recent development of small intestinal endoscopic techniques such as capsule and DBE, the diagnosis and understanding of these entities has improved. The small intestinal ulcers in CMUSE occur predominantly in the ileum; the terminal ileum is usually spared. The ulcers are usually multiple and never progress to a cobblestone appearance, fissure, or fistula formation. The ulcers are restricted to the mucosa or submucosa; they never extend to the proper muscular layer. The mucosal lesions are characterized by mixed infiltration of plasma cells, lymphocytes, and eosinophils. ${ }^{3}$ Crohn's disease, tuberculosis, malignancy, and other infectious disease were excluded in this case. ${ }^{4}$ There are no specific laboratory tests for the diagnosis of CMUSE. We could not exclude small intestinal tuberculosis in this patient with typical transverse small intestinal ulcers on DBE, despite the negative tuberculosis-specific Ag induced interferon-gamma test result. Therefore, we empirically treated the patient with antituberculosis medications for 2 months. The response to antituberculosis medication was not sufficient, thus we decided to resect the small intestinal lesions to prevent obstruction events and confirm the pathologic diagnosis.

The treatment of CMUSE remains symptomatic. Typically, CMUSE responds to glucocorticosteroids, but steroid dependence often develops, and a case of steroid-refractory CMUSE was previously reported. ${ }^{5}$ Treatment with oral 5 -aminosalicylic acid and azathioprine failed to induce mucosal healing or prevent small intestinal strictures. ${ }^{3}$ For patients with steroid dependence or refractory disease, surgical resection would be the last choice for treatment.

\section{REFERENCES}

1. Perlemuter G, Guillevin L, Legman P, Weiss L, Couturier D, Chaussade S. Cryptogenetic multifocal ulcerous stenosing enteritis: an atypical type of vasculitis or a disease mimicking vasculitis. Gut 2001;48:333-338.

2. Kohoutova D, Bures J, Tycova V, et al. Severe cryptogenic multifocal ulcerous stenosing enteritis. A report of three cases and review of the literature. Acta Medica (Hradec Kralove) 2010;53:25-29.

3. Matsumoto T, Iida M, Matsui T, Yao T. Chronic nonspecific multiple ulcers of the small intestine: a proposal of the entity from Japanese gastroenterologists to Western enteroscopists. Gastro- 
intest Endosc 2007;66(Suppl 3):S99-S107.

4. Chang DK, Kim JJ, Choi H, et al. Double balloon endoscopy in small intestinal Crohn's disease and other inflammatory diseases such as cryptogenic multifocal ulcerous stenosing enteritis
(CMUSE). Gastrointest Endosc 2007;66(Suppl 3):S96-S98.

5. Kim CW, Yu CS, Yoon YS, Yoon SN, Lim SB, Kim JC. Steroidrefractory cryptogenic multifocal ulcerous stenosing enteritis. Am J Surg 2011;202:e48-e51. 